\title{
Transición de la presencialidad a la virtualidad en las actividades de trabajo cooperativo en un taller de teatro inclusivo
}

\author{
Transition from face to face to virtuality in cooperative work activities \\ In an inclusive theater workshop
}

\section{Transição do presencial ao virtual em atividades de trabalho cooperativo em uma oficina de teatro inclusiva}

\author{
Alicia Sandoval Poveda \\ Universidad Estatal a Distancia \\ San José, Costa Rica \\ asandovalp@uned.ac.cr \\ (D) ORCID: https://orcid.org/0000-0002-9678-7586 \\ Linda Madriz Bermúdez \\ Universidad Estatal a Distancia \\ San José, Costa Rica \\ Imadriz@uned.ac.cr \\ (1) ORCID: https://orcid.org/0000-0003-2079-3987
}

Recibido - Received - Recebido: 15 / 09 / 2021 Corregido - Revised - Revisado: 29 / 10 / 2021 Aceptado - Accepted - Aprovado: 12 / 11 / 2021

DOI: https://doi.org/10.22458/ie.v24i36.3896

URL: https://revistas.uned.ac.cr/index.php/innovaciones/article/view/3896

\begin{abstract}
Resumen: La presente sistematización pretende analizar la transición del trabajo cooperativo en el Grupo de Teatro Rompecabezas, del espacio presencial al virtual, durante la pandemia por COVID-19, en un taller de teatro que tiene por objetivo desarrollar habilidades sociales en jóvenes con situación de discapacidad. Para ello, se realizó un análisis de contenido de las bitácoras y videos de las sesiones de trabajo en subgrupos de improvisación, en las que participaron 15 jóvenes con situaciones de discapacidad, además de una encuesta a estos y a las personas facilitadoras del espacio. Se encontró, que la experiencia para ajustar a la virtualidad el tipo de actividad que ya se realizaba de forma presencial, dio un rol más preponderante a la persona facilitadora para asegurar la dinámica de trabajo de los subgrupos. Las técnicas consistían en divisiones en subgrupos, con una premisa base para crear una improvisación en conjunto, ya fuera una escena o una canción, en lo presencial o una historia conjunta, en lo virtual. Se utilizó la estructura grupal del tipo informal, de hacer grupos pequeños al azar con el rol facilitador del tipo formal, que orienta a los subgrupos en el trabajo cooperativo. De los componentes de este tipo de trabajo, se destacaron la interdependencia positiva, la responsabilidad y la interacción estimulante, y se identificó la necesidad de fortalecer las habilidades sociales y el procesamiento grupal. Se identificó, la pertinencia de investigar más sobre las técnicas de trabajo cooperativo, en el medio virtual y artístico para personas en situación de discapacidad.
\end{abstract}

Palabras clave: Trabajo cooperativo, teatro, educación virtual, educación especial, educación.

\begin{abstract}
The present systematization aims to analyze the transition of cooperative work in the Rompe Puzzle Theater Group, from face-to-face to virtual space, during the COVID-19 pandemic, in a theater workshop that aims to develop social skills in young people with disabilities. For this, a content analysis of the logs and videos of the work sessions in improvisation subgroups was carried out, in which 15 young people with disabilities participated, in addition to a survey of these and the people facilitating the space. It was found that the experience to adjust to virtuality, the type of activity that was already carried out in person, gave a more preponderant role to the facilitator to ensure the work dynamics of the subgroups. The technique consisted of subgroups and a basic premise to improvise together in the virtual session-whether a scene or a song, in person, or a joint story. A group structure of the informal type was used, making small groups randomly facilitating the role of the formal type, which guides the subgroups in cooperative work. Some of the components of this type of work were: positive interdependence, responsibility, and stimulating interaction were highlighted, and the need to strengthen social skills and group processing was identified. The relevance of investigating more about cooperative work techniques in the virtual and artistic environment for people with disabilities was identified.
\end{abstract}

Keywords: Cooperative work, theater, virtual education, special education, education. 
Resumo: A presente sistematização analisa a transição do trabalho cooperativo no Grupo Rompe Puzzle de Teatro, do espaço presencial para o virtual, durante a pandemia COVID-19, em uma oficina de teatro que visa desenvolver habilidades sociais em jovens com deficiência. Por tanto, uma análise de conteúdo dos logs e vídeos das sessões de trabalho foi realizada em subgrupos de improvisação, da qual participaram 15 jovens com deficiência, além de uma sondagem sobre eles e as pessoas facilitadoras do espaço. Corroborou-se que a experiência de adequar à virtualidade ao tipo de atividade que já era realizada presencialmente, conferiu ao facilitador um papel ainda mais preponderante para poder garantir a dinâmica de trabalho dos subgrupos. As técnicas consistiam em divisões em subgrupos, tendo como premissa básica criar uma improvisação virtual em conjunto, de uma cena ou uma música, ou uma história conjunta presencial. Foi utilizada a estrutura grupal do tipo informal, de formação de pequenos grupos aleatórios com o papel facilitador do tipo formal, que orienta os subgrupos no trabalho cooperativo. Dos componentes desta categoria de trabalho destacouse a interdependência positiva, a responsabilidade e o estímulo à interação, e identificou-se a necessidade de fortalecer as habilidades sociais e o processamento grupal. Identificou-se a relevância de se fazer mais pesquisas sobre técnicas de trabalho cooperativo, no ambiente virtual e artístico para pessoas com deficiência.

Palavras-chave: Trabalho cooperativo, teatro, educação virtual, educação especial, educação.

\section{INTRODUCCIÓN}

La situación de pandemia por el virus responsable de la COVID-19, generó una serie de cambios en los procesos educativos, al afectar la posibilidad de la educación presencial (Hurtado, 2020), lo que llevó a nuevos planteamientos para la educación artística, desde el ámbito virtual (Huauya, 2021). El Grupo Rompecabezas es un espacio de formación teatral inclusivo, que busca el desarrollo de habilidades sociales en jóvenes, en situación de discapacidad. Este grupo trabajó de manera presencial desde el año 2016; sin embargo, en el año 2020 se suspendió el trabajo presencial y la formación artística, a raíz de la pandemia.

La población en situación de discapacidad, está más expuesta durante el periodo de pandemia, a enfrentar retos a causa de la discriminación (Huete, 2020). Por esta razón, durante el año 2020, se dedicó el trabajo del Grupo Rompecabezas a dar apoyo y acompañamiento, ante la situación de pandemia (Sandoval-Poveda y Madriz-Bermúdez, 2020). Sin embargo, otro de los riesgos durante una pandemia, es que estas personas dejen de recibir la atención que necesitan (Pellicano y Stears, 2020). Por esta razón, se consideró necesario en 2021, retomar el trabajo de teatro, pero ahora en un medio virtual. Para ello, era necesario mantener los trabajos de tipo cooperativo que se utilizaban, para fomentar el aprendizaje y el desarrollo de habilidades sociales. Por tanto, la transición al modelo virtual, implicaba dar respuesta al problema de cómo realizar la transición de actividades cooperativas presenciales a otras de tipo virtual.

Para orientar esta experiencia, se consultaron algunas anteriores, en las cuales se había establecido, que el trabajo cooperativo puede ayudar al desarrollo de procesos emocionales y habilidades sociales e interpersonales (Jones y Doolittle, 2017). En el área artística, Pérez-Aldaguer (2013) encontró que hubo diferencias significativas favorables, en las variables de competencia social, clima grupal y habilidades sociales en una experiencia de teatro musical.

En el caso de la población en situación con discapacidad, se encontró que el trabajo cooperativo aumenta la capacidad de trabajo, la adquisición de conocimientos y la motivación, cuando el estudiantado percibe que todos tienen tareas igual de importantes, se evalúa el progreso de cada estudiante por separado y no se hacen comparaciones (Temprado Bernal, 2009). También ha mostrado ventajas, como promover el respeto entre los pares y promover las relaciones interpersonales, fortalecer el mantenimiento del grupo, mejorar la aceptación de las otras personas, afrontar sus demandas y hacer amistades (SchwarzAguayo, 2011), y en general, favorece el desarrollo de la interacción social (Silva Cruz, 2018). El trabajo cooperativo evidencia un clima escolar favorable y una influencia positiva, en la enseñanza de personas en situación de discapacidad, creando conocimientos desde sus propias habilidades, destrezas y valores (Cedeño-Muñoz \& Barcia-Briones, 2020). En el caso de personas diagnosticadas con Trastorno del Espectro Autista, se ha encontrado que las sesiones de trabajo cooperativo en 
edad preescolar, pueden aumentar la atención con respecto a las sesiones de trabajo individual (Segui y Durán, 2011), también que puede promover la interacción social y mediar las expectativas de trabajar con pares (Peters et al., 2013).

Sin embargo, debe considerarse que en todos estos trabajos, se realizaron intervenciones de aprendizaje cooperativo, en el contexto de clases presenciales (Herrada Valverde y Baños Navarro, 2018). Hay menos trabajos, con respecto al desarrollo de actividades de trabajo cooperativo en ambientes virtuales. Sin embargo, la atención de personas en situación de discapacidad a través de medios virtuales se ha visto potenciada durante la situación de pandemia (Pellicano y Stears, 2020). Incluso antes de esta crisis, se valoraba el medio virtual como una ventaja, porque propicia interacciones entre docentes y estudiantes, mientras aprenden a su ritmo, según su habilidad y de forma activa (Joan, 2018).

A partir de este contexto, la presente experiencia tomó como referente conceptual, que el trabajo cooperativo se deriva de las teorías de aprendizaje cooperativo, donde se plantea el uso de grupos pequeños de estudiantes, que trabajen juntos para maximizar el aprendizaje (Butera y Buchs, 2019). La cooperación tiene cinco componentes: el primero, es la interdependencia positiva, la cual se refiere a que haya una meta común que solo puede alcanzarse, si todas las personas en el grupo lo logran juntas. El segundo, es la responsabilidad y rendición de cuentas personal, en el cual todas las personas tienen la misma meta, son responsables unas por otras, por repartirse el trabajo y ayudarse mutuamente. En tercer lugar, la interacción estimulante, en la que se intercambian recursos, construyen un discurso común, discuten y se apoyan en sus pares. En cuarto lugar, las habilidades sociales, las cuales se desarrollan de manera constructiva y centradas en la tarea, la comunicación precisa, la discusión y la confrontación. En quinto lugar, el procesamiento grupal, implica reflexionar sobre las acciones, decisiones, comunicación y desempeño como grupo, lo que les permite reconsiderar decisiones y generar cohesión, e identidad de grupo (Butera y Buchs, 2019).

El trabajo cooperativo puede ser de tipo formal, en el cual los grupos tienen que completar una tarea juntos; de tipo informal, con grupos que se reúnen solo unos minutos para una actividad o de tipo grupos de base cooperativa que son permanentes, durante un periodo. (Johnson y Johnson, 2014a).

El trabajo cooperativo tiene resultados positivos, como el esfuerzo para alcanzar logros, relaciones interpersonales positivas y ajuste psicológico. Las personas aprenden mejor a preocuparse por otras, cuando trabajan de forma cooperativa en lugar de competitiva. Trabajar de forma cooperativa con pares, beneficia la salud psicológica y aumenta la autoestima, independencia, autoconfianza y autonomía. Al trabajar en equipo, se debe interactuar con los demás, mejorando las habilidades y competencias sociales, y potenciando las relaciones (Johnson y Johnson, 2014b). La cooperación va a tener un rol vital en construir relaciones positivas, tanto en el trabajo cara a cara, como en el que se realice en medios virtuales. En este segundo tipo, se debe considerar que las relaciones on-line se construyen con un propósito común, son relaciones tan reales como las demás y permiten un alcance sin límites geográficos, entre personas (Johnson y Johnson, 2014a).

Esta sistematización tiene por objetivo, analizar la transición de presencialidad a virtualidad, de las actividades de trabajo cooperativo del taller de teatro inclusivo, del Grupo Rompecabezas. Se establecieron como objetivos específicos, describir las técnicas de trabajo cooperativo de los talleres presenciales 2019 y los talleres virtuales 2021, identificar la manera en que las técnicas utilizadas propician los componentes del trabajo cooperativo, describir el tipo de trabajo cooperativo utilizado, indagar la percepción de las personas participantes del grupo con respecto a las actividades de trabajo cooperativo, y comparar las técnicas que se pusieron en práctica, en ambos años, para propiciar el trabajo cooperativo. 
La experiencia recopilada se enmarcó en el proyecto de investigación-acción, llamado "Desarrollo de habilidades sociales para jóvenes con trastorno del espectro autista, a través de una experiencia artística: el teatro". De los años 2016 a 2019, se trabajó de manera presencial en talleres de teatro semanales. A inicios de 2020, con motivo de la pandemia, fue necesario suspender los talleres, priorizando la atención emocional de la emergencia, pero se retomaron de manera virtual en 2021. Con el objetivo de analizar la transición del trabajo cooperativo, del espacio presencial al virtual, se realizó un análisis de contenido de los videos y bitácoras de las sesiones de trabajos, en subgrupos del año 2019 (talleres presenciales) y el año 2021 (talleres virtuales). No se estudió el año 2020, porque en este no se realizaron sesiones de teatro, ni ejercicios en subgrupos. A su vez, se realizó una indagación entre las personas participantes y personas facilitadoras de ambos espacios.

Se seleccionó trabajar sobre las bitácoras y videos de ambos periodos, para poder analizar los métodos de trabajo cooperativo, utilizados en subgrupos de trabajo. No se consideraron las sesiones de montaje o ensayo de obras de teatro completas, sino los momentos de formación actoral, específicamente los de trabajos en subgrupos para improvisación, ejercicio que se realizó de forma presencial en 2019, y en el año 2021 de forma virtual. Los videos de 2019 consistían en 21 cortos, de entre 30 segundos a 1 minuto cada uno, con las presentaciones improvisadas. Los videos de 2021, consistían en las presentaciones en Zoom $^{\oplus}$ de historias improvisadas. Los videos de la etapa presencial fueron realizados en el teatro, donde recibían las sesiones de formación teatral. Los videos de la etapa virtual, se realizaron en la plataforma de videoconferencia Zoom ${ }^{\circledast}$. Las bitácoras de ambos años, consisten en textos escritos con las opiniones y observaciones de todas las personas del equipo investigador, después de cada sesión. Para realizar este análisis de contenido se partió de tres grandes categorías: técnicas de trabajo, tipo de trabajo cooperativo utilizado y componentes del trabajo cooperativo. Cada categoría, incluía una serie de componentes para analizar, como se puede apreciar en la Tabla 1.

TABLA 1

Componentes de las categorías de análisis de la información

\begin{tabular}{cc}
\hline \multicolumn{1}{c}{ Categoría } & Componentes de la categoría \\
\hline Técnicas de trabajo en las sesiones & $\begin{array}{c}\text { Técnicas de aprendizaje presencial } \\
\text { Técnicas de aprendizaje virtual }\end{array}$ \\
\hline Tipo de trabajo cooperativo utilizado & Tipo formal \\
Tipo informal \\
Tipo grupos base
\end{tabular}

Fuente: Elaboración propia.

Con estas categorías y sus componentes definidos, se analizaron las bitácoras y los videos en las tres categorías anteriores, y se sistematizaron en una matriz de información. Con base en la matriz, se comparó para cada componente del trabajo cooperativo, cuáles técnicas se utilizaron en lo presencial y lo virtual. A partir de esta comparación, se procedió a identificar y describir las adaptaciones que se identificaron en la transición de lo presencial a lo virtual, y finalmente, analizar los resultados de esta adaptación. De igual forma, se definió para ambos tipos de modalidad, qué tipo de trabajo cooperativo se utilizó.

Para garantizar la validez y confiabilidad, se triangularon los datos del análisis de contenido con dos formularios, por medio de la plataforma MS Forms ${ }^{\oplus}$; uno, dirigido a las personas que habían facilitado los 
trabajos en subgrupos de ambos años y otro, dirigido a las personas participantes. Ambos formularios se detallan a continuación. El primero, dirigido a personas facilitadoras, consistía en dos escalas Likert para valorar, en las actividades presenciales y las virtuales respectivamente, la frecuencia con la que se presentaban en los subgrupos, conductas relacionadas con el trabajo cooperativo. Además, se les preguntaba por similitudes y diferencias entre el trabajo presencial y virtual. Por su parte, las personas participantes contestaron un instrumento de ocho ítems donde se les pedía, en preguntas cerradas, valorar en una escala de uno a cinco, los diferentes tipos de actividades de trabajo cooperativo realizadas (escenas, música e historias); además, debían decir si estaban de acuerdo o no con que en los trabajos en subgrupos, se cumpliera una frase para cada componente del trabajo cooperativo y, en dos preguntas abiertas, debían opinar sobre qué les gustaba y qué no, de trabajar en subgrupos. Estos resultados se contrastaron con los del análisis de contenido realizado.

\section{Técnicas de trabajo cooperativo analizadas}

Se analizaron los ejercicios de improvisación orientados a propiciar el trabajo cooperativo en ambos años. En la modalidad presencial, hubo dos ejercicios de este tipo. El primero, era la improvisación de escenas, en la cual se dividía al grupo en subgrupos de entre 4 o 5 personas. Cada subgrupo contaba con una persona facilitadora que apoyaba el desarrollo de la actividad. Se les daba una premisa para que entre 15 o 30 minutos, elaboraran una breve escena que actuar frente al grupo. Una vez terminado el tiempo, se hacía la presentación frente al resto del grupo y recibían retroalimentación de la docente de teatro. En siete sesiones del año 2019, se realizó este tipo de ejercicios. El segundo tipo de actividad de improvisación, consistía en trabajar con las canciones de la obra de teatro en montaje ese año. Se procedía igual, pero en lugar de una premisa, se le asignaba la letra de una canción de un texto teatral y, se les pedía improvisarle melodía, ritmo y coreografía.

Dentro de las actividades virtuales ejecutadas en la primera mitad del año 2021, se realizaron cinco sesiones centradas en la improvisación. La primera de ellas, consistió hablar de qué es una secuencia y luego, construir una historia conjunta con el método de "si mágico". La segunda, consistió en dividir el grupo en subgrupos. Cada uno leyó el mismo cuento sin final y cada persona tenía que aportar ideas, para decidir en subgrupo cómo les gustaría que terminara. Cada subgrupo trabajó en una sala de Zoom ${ }^{\circledast}$ ("breakout room") con una persona facilitadora por 20 minutos y después, en plenaria, cada grupo expuso su final. Las otras tres sesiones, se trabajaron en construir una historia con una premisa diferente por subgrupo. En la primera ocasión, la premisa era un escenario, un personaje y una situación; en la segunda, una frase y en la tercera, dos sonidos. La dinámica era la misma: se trabajaba en subgrupos, con la instrucción de que cada persona debía aportar y con la moderación de un facilitador. El subgrupo decidía cómo iban a presentar la historia, de forma que todas las personas participaran y después le exponían al resto del grupo.

Al analizar el trabajo realizado, se evidencia que todas estas técnicas, tanto presenciales como virtuales, se realizaron con un tipo de trabajo cooperativo informal, dado que los subgrupos se formaron en cada sesión, con las personas participantes presentes, intentando que no se repitieran las agrupaciones de la semana anterior. Una vez formado el grupo, se les asignaba la tarea del día y se disolvía el grupo al terminar. Sin embargo, también es posible apreciar que el rol de la persona facilitadora, fue de una intervención constante para orientar el trabajo cooperativo, lo que suele relacionarse más con el tipo formal, pero en este caso se hizo necesario.

\section{Componentes del trabajo cooperativo}


A continuación, se presenta la forma en que estas actividades, presenciales y virtuales, propiciaron los componentes del trabajo cooperativo. El primero, es la interdependencia positiva. Se encontró que, al determinar desde la instrucción de que todas las personas del subgrupo tenían que participar, nadie podía quedarse sin hacerlo, incluidas las personas que en otro tipo de actividades participaban menos. En la presencialidad, se destacaron los siguientes aspectos: si alguna persona intentaba alejarse, alguien del equipo facilitador le desplazaba de regreso con el grupo, el grupo no podía presentar si le faltaba una persona y, se detenía a cualquier persona que quisiera empezar sin el resto del grupo. Cuando alguien llegaba tarde, solicitaba que se le asignara subgrupo, contaban cuántas personas tenían en el subgrupo para decidir lo que harían, mostrar alarma si algún participante debía retirarse antes de la presentación, llamar a la persona que se quedaba sentada cuando iban a participar, planear en conjunto una coreografía y respetarla (incluso por parte de aquellas personas que no les gustaba bailar). En el espacio virtual, fue necesaria una mayor mediación por parte de las personas facilitadoras, que en la modalidad presencial, en este lugar fue necesario que la persona facilitadora interviniera más para dar la palabra a la persona que le correspondía, abrir el micrófono y participar, y se hizo necesario que se indicara cuando faltaba alguien y se mediara en la repartición de tareas. Todos los subgrupos optaron por contar las historias entre todos $y$, cuando alguien olvidaba su parte, otra persona del subgrupo le ayudaba.

En cuanto a la responsabilidad y rendición de cuentas, esta se propiciaba en las actividades, al establecer la regla de que toda persona del subgrupo debía participar, sin importar sus gustos o su capacidad para aprender texto, cantar canciones o inventar historias. La persona facilitadora también les recordaba sus roles, cuando era necesario. En la presencialidad, se procuraba desde la facilitación, asegurarse que una persona que no tenía habilidad para decir texto o cantar, tuviera un rol asignado, ya fuera actuando o con un instrumento de percusión, lo que les daba una responsabilidad con el grupo. Fue posible apreciar que, a pesar de sonrojarse o esconderse durante las canciones o las presentaciones, las personas seguían participando. Incluso en casos que la persona se negaba a bailar, se mantenía de pie junto a su grupo en la presentación. Una vez terminada su participación, algunas personas se alejaban físicamente del resto de integrantes del grupo, evidenciando que se había mantenido a su lado, por ser parte del subgrupo.

Por su parte, en la virtualidad, fue necesario desde la moderación del espacio, insistir en que todas las personas del subgrupo tenían que participar, y que tuvieran responsabilidades asignadas durante la presentación de las historias, ya fuera hacer sonidos o decir frases. Algunas personas asumieron responsabilidades de forma autónoma, mientras que a otras, fue necesario asignarles su participación. Al contrario de las presentaciones presenciales, nadie presentaba a la vez (todos bailando al mismo tiempo, por ejemplo), sino que a cada persona le correspondía en su momento, intervenir desde su propia cámara y micrófono. Se destacaba que participantes que, en la forma presencial, participaban poco o se refugiaban detrás del resto, en las sesiones virtuales se esforzaron para hacer su parte, en el momento que les correspondió.

En cuanto a la interacción estimulante, cada persona del grupo aportara desde sus talentos e intereses, lo que en ocasiones correspondía a la persona facilitadora, asegurarse que ocurriera. En la presencialidad, quienes sabían más de música les explicaban a los demás, quienes memorizaban más texto tenían más líneas, los que hablaban poco acompañaban con gestos; quienes no cantaban, tocaban instrumentos o bailaban, según su preferencia. Fue posible observar cómo celebraban, cuando se les decía con quiénes iban a trabajar, chocando palmas o aplaudiendo, motivándose a trabajar como subgrupo, sin que mediara facilitación docente para esas expresiones. Por su parte, en la virtualidad, en cada subgrupo, se presentaron muchas ideas y cada persona aportó estilos diferentes para narrar frente al grupo sus finales o sus historias. En algunos casos, incluso llegaban a formular varios finales de cuentos o varias historias. Además, a la hora de repartirse las responsabilidades para presentar, algunos sugerían quién de las personas del subgrupo podía encargarse de narrar cierta parte o de hacer ciertos sonidos. Cuando 
a alguna persona se le dificultaba aportar ideas, otras le daban opciones. Era necesario, de parte de la persona facilitadora, dar la palabra para que no se extendiera demasiado una sola intervención, así como estimular el inicio de la discusión.

En cuanto a las habilidades sociales, el planeamiento de las improvisaciones siempre estuvo realizado a través del diálogo. La persona facilitadora tenía que participar en mayor o menor medida, según el caso, pero cada participante debía aportar ideas, manifestar si estaba de acuerdo con lo que se proponía o no, expresar lo que le gustaba y lo que no. En lo presencial, lo hacían de forma verbal, gestual o musical. Además, en la presentación, también usaban comunicación no verbal para indicarse mutuamente a qué lado del escenario moverse o cuando a alguien le tocaba su turno si se le había olvidado.

Por su parte, en lo virtual, el diálogo fue mediado siempre por la persona facilitadora, pero en general, se mantuvo un ambiente de respeto para las ideas de cada quien y se corrigió, cuando alguna persona querría interrumpir. El trabajo en subgrupos permitió, que todas las personas del grupo participaran desde sus posibilidades y aprendieran a seguir con su intervención, lo dicho previamente por las otras personas, de manera que su historia tuviera una secuencia. Algunas personas participantes abrían y cerraban su micrófono por iniciativa propia y a otras, se les tenía que indicar. Fue un ejercicio de aprender tolerancia para esperar a que la intervención de alguien terminara, para poder pedir la palabra. En el caso de personas con problemas de pronunciación, se les permitía insistir hasta darse a entender, dado que era más limitado el uso de la comunicación gestual, limitada al rango de la cámara. Cuando el subgrupo terminaba y quedaba tiempo en la sala aparte, aprovechaban para hablar de otros temas.

Finalmente, en cuanto al procesamiento grupal, en lo presencial, después de cada presentación se debatía en plenaria y la docente brindaba retroalimentación, sobre los principios de actuación y cómo se aplicaban a lo que se había hecho. Las mismas personas del subgrupo podían identificar errores que habían cometido, como darle la espalda al público en algún momento, o hablar muy bajo, y recibían correcciones de la docente de teatro. Algunos intentaban disculparse por los errores y otros, mostraban resistencia a recibir retroalimentación. Más allá de las correcciones, mostraban orgullo del trabajo presentado por medio de sonrisas, exclamaciones sobre su buena actuación, choques de manos o puños. En lo virtual, no se hizo una reflexión por cada subgrupo, pero después de cada presentación recibían el aplauso del resto del grupo y se manifestaban con mayor alegría y motivación, que en sesiones que no había trabajos en subgrupo.

\section{Apreciación de personas participantes y facilitadoras}

Se pidió a las personas participantes, valorar de 1 a 5 (siendo 1 nada y 5 lo máximo), las actividades en subgrupos, cuando tenían que improvisar una escena y una canción de forma presencial, y cuando tenían que improvisar historias en Zoom ${ }^{\circledast}$. Para la improvisación de escenas, el promedio de la calificación brindada fue de 4,77, el de las canciones fue de 4,54 y para las actividades virtuales en subgrupos fue de 4,13.

Posteriormente, se les presentaron cinco enunciados, cada uno relacionado con uno de los componentes del trabajo cooperativo, para que dijeran si les parecía que eran ciertos al trabajar en subgrupos en el teatro. De igual forma, se les presentaron los mismos cinco enunciados para que dijeran si les parecía que eran ciertos al trabajar en el Zoom ${ }^{\oplus}$. En la Tabla 2 se presentan los resultados de ambas consultas, que corresponde al porcentaje de participantes del grupo que concuerda sobre cada enunciado. Cada enunciado corresponde a uno de los componentes del trabajo cooperativo, y lo que valoraron fue si lo dicho en cada frase, se presentaba en la modalidad presencial y en la modalidad virtual. 
TABLA 2

Porcentaje de participantes que concuerdan en que las afirmaciones relacionadas con cada componente del trabajo cooperativo son ciertas para el trabajo presencial en el teatro en subgrupos

\begin{tabular}{|c|c|c|c|}
\hline Componente & Afirmación & $\begin{array}{c}\text { Porcentaje de } \\
\text { participantes que } \\
\text { concuerdan con que la } \\
\text { frase se cumple en el } \\
\text { trabajo presencial }\end{array}$ & $\begin{array}{c}\text { Porcentaje de } \\
\text { participantes que } \\
\text { concuerdan con que la } \\
\text { frase se cumple en el } \\
\text { trabajo virtual }\end{array}$ \\
\hline Interdependencia grupal & $\begin{array}{l}\text { Todos tenemos que participar } \\
\text { para que el trabajo salga bien }\end{array}$ & $86 \%$ & $86 \%$ \\
\hline $\begin{array}{l}\text { Responsabilidad rendición } \\
\text { de cuentas }\end{array}$ & $\begin{array}{l}\text { Tengo que hacer mi parte bien } \\
\text { para que la presentación quede } \\
\text { bien }\end{array}$ & $86 \%$ & $80 \%$ \\
\hline Interacción estimulante & $\begin{array}{l}\text { Todos tenemos cosas diferentes } \\
\text { que aportar }\end{array}$ & $73 \%$ & $73 \%$ \\
\hline Habilidades sociales & $\begin{array}{l}\text { Podemos hablar mucho para } \\
\text { ponernos de acuerdo }\end{array}$ & $46 \%$ & $53 \%$ \\
\hline Procesamiento grupal & $\begin{array}{l}\text { Cuando terminamos la presenta- } \\
\text { ción podemos celebrarlo como } \\
\text { grupo }\end{array}$ & $86 \%$ & $93 \%$ \\
\hline
\end{tabular}

Fuente: Elaboración propia.

Los resultados no muestran una mayor diferencia entre ambas modalidades; sin embargo, destacó que había más personas para las cuales, en la virtualidad se puede hablar mucho para ponerse de acuerdo, lo que corresponde con el hecho de que se media para que todas las personas participen y todo pasa por el diálogo. Además, un porcentaje mayor creía que se celebra más como grupo en la virtualidad y un porcentaje menor creía que tenía que hacer bien su parte para que la presentación quedara bien, lo que resulta llamativo porque en este medio tengan que intervenir por separado; sin embargo, si alguien se equivocaba, el resto del subgrupo le ayudaba o corregía.

Con respecto a lo que les gusta de trabajar en subgrupos, en las respuestas se destacó el aspecto lúdico, la oportunidad de hablar y compartir, aportar ideas y que todos participen. Se criticó que algunas personas del grupo hablan mucho y hacen ruido, y una persona indica que no le gusta trabajar virtual.

Por su parte, con las personas facilitadoras, se indagó la frecuencia de conductas relacionadas con el trabajo cooperativo en los trabajos de improvisación en ambas modalidades. Estas concuerdan en que, en el trabajo presencial, la mayoría de las veces los y las participantes se preocupaban de que todas las personas del grupo participaran, cada participante aportaba conocimientos, intereses y talentos propios, todas las personas tenían una tarea que realizar para completar el trabajo y al terminar, el subgrupo se felicitaba por el resultado. Al contrario, solo en ocasiones asumían la responsabilidad de aportar, sin que se les tuviera que instigar, intercambiaban ideas y propuestas sin necesidad de mediación, o se mostraban críticos con el resultado de su trabajo. Además, era poco frecuente que negociaran cuál de las ideas propuestas se iba a quedar, sin que la persona facilitadora tomara la decisión final. De esta forma, en las actividades presenciales, si bien había componentes de interdependencia grupal e interacción estimulante, tanto las habilidades sociales de comunicación como la asignación de responsabilidades, eran mediadas por la persona facilitadora. En cuanto al procesamiento grupal, el subgrupo disfrutaba su presentación y la celebraba, pero sin mostrar criticidad hacia su proceso.

En cuanto a la facilitación de actividades virtuales, se destacó que en el medio virtual era más frecuente, la necesidad de mediación pedagógica de la persona facilitadora para visibilizar que todas las personas necesitan participar, para completar la tarea, pero cada participante asumía mayor responsabilidad de 
lo que le toca hacer. Sin embargo, la mediación necesaria por parte de las personas facilitadoras para negociar cuáles ideas se utiliza y que cada persona haga su aporte era igual que en el medio presencial, lo que aumentó fue la necesidad de una persona que brinde la palabra para que se asignara quién podía abrir el micrófono. El subgrupo se mostró más crítico con sus resultados en la forma virtual y celebraban más al concluir sus presentaciones.

Según la apreciación de las personas facilitadoras, en ambos medios, presencial y virtual, hay una ruta clara y objetivos de lo que se va a realizar, es necesario pedir concentración constantemente, y las personas participantes muestran una buena actitud hacia el trabajo, haciendo aportes. Se diferencia el trabajo en ambos medios, en que en el espacio presencial hay menos distractores que los que tiene cada persona desde su hogar, el espacio que se puede utilizar es solamente el que esté frente a la cámara y la interacción es más limitada.

\section{Análisis de la transición del medio presencial al virtual}

Por medio del análisis de las bitácoras, los videos de actividades y la retroalimentación de participantes y personas facilitadoras, se pretendió analizar la transición del trabajo presencial al virtual en cuanto a las actividades de trabajo cooperativo. En la Tabla 3, se presentan las prácticas identificadas a través del análisis, como aquellas que permitieron la transición de lo presencial a lo virtual del trabajo cooperativo.

TABLA 3

Resumen de las técnicas de transición de lo presencial a lo virtual para conservar el trabajo cooperativo

Prácticas de transición

1. Mantenimiento de la estructura de trabajo adaptada a lo virtual persona facilitadora trabajo

4. Buscar alternativas para la participación integral, según habilidades

\section{Presencial}

Se distribuyen los subgrupos por el teatro. Se realiza la presentación en un escenario. La instrucción pide aportes de cada participante.

Ayuda en el proceso de discusión y toma de decisiones, y a centrarse en la premisa de trabajo. Facilita el diálogo, hace respetar turnos y da guías que le permitan al grupo terminar su tarea.

Aproximadamente 30 minutos de trabajo en subgrupos.

Las personas con dificultades de dicción, memoria o lectura usaban lenguaje corporal y en las canciones, tocaban instrumentos de percusión.

Grupos de hasta seis personas

5. Propiciar la comunicación en subgrupos pequeños

6. Énfasis en el proceso y no en el resultado
Atención a las técnicas actorales utilizadas en la presentación final de la improvisación.

\section{Virtual}

Se retiran a salas de trabajo virtual separadas.

Se realiza la presentación en la sesión principal de la videollamada.

Es la misma instrucción.

Acompaña a los subgrupos en salas virtuales separadas.

Se suma a su rol, el manejo de dar el turno para abrir el micrófono, a fin de mantener el orden y que toda persona tenga turno para participar.

Se redujo a 15 o 20 minutos de trabajo en subgrupos.

Las personas con dificultades de dicción o memoria intervienen con la creación de sonidos de animales o efectos de sonido (como una sirena o el toque de una puerta).

Grupos de máximo cinco personas (en muchos casos de 4)

No se evaluó el contenido, complejidad o secuencia de las historias creadas. Se dio énfasis al proceso creativo.

Fuente: Elaboración propia.

En primer lugar, se encuentra que, si bien cambió el medio de ejecución, la estructura central de estas actividades se mantuvo, lo que dio estabilidad y seguridad a las personas participantes. El hecho de usar una dinámica de trabajo familiar a las realizadas anteriormente, en el medio presencial, facilitó a las 
personas participantes adaptarse a realizar una actividad conocida en un medio nuevo. Según Johnson y Johnson (2014b), este tipo de trabajo con la premisa de que hay una sola meta en común, en la que deben participar todos para alcanzarla potencia la interdependencia social, lo que ocurrió según lo que fue posible identificar en ambas modalidades de trabajo, de igual forma que al parecer, el hecho de que todas las personas contribuyeran en el resultado final contribuye a la interdependencia positiva (Butera y Buchs, 2019).

En segundo lugar, se debe rescatar la importancia de la persona facilitadora en el desarrollo del trabajo en subgrupos. Según Johnson \& Johnson (2014), en la ejecución de los ejercicios en subgrupos, desde el trabajo cooperativo formal, este rol incluye dar instrucciones, previo a la realización de la tarea, explicar cómo será la estructura cooperativa para llevarla a cabo, y monitorear con el fin de mejorar el trabajo que realizan los grupos y su efectividad, lo que coincide con la forma en que se ejerció este rol en ambas modalidades de trabajo, según esta experiencia. La importancia del rol facilitador ha sido destacado en otros casos, como alguien que guíe el trabajo autónomo, dé instrucciones claras y brinde apoyo en las actividades (Koris \& Vuylsteke, 2020), así como se ha destacado su necesidad en las actividades de trabajo cooperativo, con la participación de estudiantes en situación de discapacidad (Temprado Bernal, 2009), y la adaptación de la práctica docente para el trabajo con estas personas, desde la virtualidad (Deliyore Vega, 2021). La retroalimentación de la persona a cargo, ayuda a potenciar la responsabilidad de cada participante, dado que reconoce y celebra sus esfuerzos, brinda apoyo a quien lo necesita y, reasigna responsabilidades para asegurarse que cada aporte sea diferente y valioso, en el resultado final (Johnson y Johnson, 2014a), lo que se visualiza en esta experiencia, cuando la intervención de la persona facilitadora asegura la participación de todo el subgrupo. Tal y como establecen Butera y Buchs (2019), desde este rol se buscan las condiciones apropiadas para promover la cooperación.

En tercer lugar, fue beneficioso disminuir el tiempo de trabajo en los subgrupos, dado que todos los grupos terminaron en menor tiempo al asignado y se empezaron a dispersar. Las adaptaciones de disminuir el tiempo de actividades a la virtualidad, en modalidades sincrónicas, han sido nombradas por otros autores (Sumardi, 2020).

En cuarto lugar, al ser una dinámica de trabajo cooperativo, era importante involucrar a todas las personas participantes como estudiantes activos (Joan, 2018), independientemente de sus capacidades, de manera que su intervención fuera necesaria para construir la improvisación, lo que se hizo, asegurando desde el espacio virtual, que las personas con problemas de dicción y memoria pudieran participar del planeamiento y presentación. Este tipo de apoyo con personas en diversas situaciones de discapacidad favorece el desarrollo de habilidades de interacción, la regulación de comportamiento, la capacidad de ayudar, controlar impulsos y tratar con un grupo de pares (SchwarzAguayo, 2011), contemplando que desde la formación artística se prioriza qué es capaz de hacer cada persona (Orr, 2010) y en el trabajo cooperativo es necesario que cada quien deba responder frente al resto del grupo con sus aportes (Butura y Buchs, 2019).

En quinto lugar, propiciar la comunicación en subgrupos de tipo informal, de tamaño no mayor a cinco personas, permitió trabajar las habilidades sociales, dado que les dio más oportunidades de hablar que las que habría tenido en el plenario con todo el grupo. Además, al estar solos en el subgrupo, desde la seguridad de la casa y tener asegurado su turno para hablar, repercutió en que algunas personas participaran más desde el medio virtual de lo que hacían en el medio presencial. Esta modalidad de trabajo, repetida y clara, favorece el desarrollo de habilidades sociales, como señalan Johnson y Johnson (2014a): estas habilidades se pueden potenciar, al ser específico con lo que cada persona debe hacer, empezando con aspectos pequeños y una habilidad a la vez, en este caso en los subgrupos para expresar ideas y tomar decisiones en grupo. Esto último, según destacan Butera y Buchs (2019), es importante aprenderlo al enfocarse siempre, en la tarea o meta a cumplir, como era en este caso, lograr la improvisación solicitada. 
En sexto lugar, el énfasis en las actividades, estuvo siempre en el proceso y no en el resultado del trabajo del subgrupo. El equipo facilitador se centró, durante cada sesión, en la dinámica de los subgrupos, motivando la creatividad grupal, la participación de todas las personas y el proceso de creación conjunta, de manera que como subgrupo pudieran sentir orgullo de la historia creada, independientemente de la calidad narrativa de esta. Además, se rescató el trabajo teatral como una forma de promover el dinamismo, la libertad, la responsabilidad y la comunicación interpersonal (Guerrero, 2014).

En resumen, la transición a la virtualidad del trabajo cooperativo, se logró con estas adaptaciones, tanto por lo analizado en las bitácoras como por lo reportado por participantes y personas facilitadoras. De acuerdo con lo presentado por algunos autores, se puede resaltar que la virtualidad elimina las barreras de distancia física y favorece la aplicación de estrategias metodológicas no tradicionales (Herrada y Baños, 2018).

\section{SÍNTESIS Y REFLEXIONES FINALES}

La pandemia provocada por el virus responsable de la COVID-19, obligó a la reinvención de los proyectos, y la migración hacia la virtualidad que tuvo que realizar el Grupo de arte inclusivo Rompecabezas, es ejemplo de esto. En un primer momento, incierto y preocupante, la organización, innovación y trabajo de equipo fueron claves, desde un repensar constante y participativo de las personas facilitadoras, con el propósito de evitar que la vulnerabilidad del grupo, se acrecentara por el distanciamiento social y, al dejar de recibir la atención que hubieran recibido, en otras condiciones desde el grupo.

En esta transición, se buscó generar técnicas de trabajo cooperativo, por medio de las cuales, se preservara la participación de todas las personas en este nuevo ambiente. Las prácticas que favorecieron esta transición fueron: conservar la estructura de trabajo en subgrupos para improvisación, potenciar el rol de la persona facilitadora del subgrupo para propiciar los elementos del trabajo cooperativo, disminuir el tiempo de trabajo en los subgrupos para adecuarse al espacio virtual, realizar adaptaciones de las formas de participación para las personas con dificultades de dicción o memoria, propiciar la comunicación en subgrupos pequeños y, mantener el énfasis de cada actividad en el proceso, no en el resultado.

Al analizar la ejecución de estas técnicas de trabajo cooperativo, se encontró una combinación de la modalidad informal (grupos para trabajos de pocos minutos, en una única sesión), con el rol activo docente de la modalidad formal, en la figura de la persona facilitadora (instrucciones claras, asignación de roles, supervisión de los subgrupos, intervención para orientar el trabajo cooperativo). De los cinco componentes del trabajo cooperativo, fue posible identificar que las premisas utilizadas y la mediación realizada facilitaron la interdependencia positiva, la responsabilidad y la interacción estimulante. El componente de habilidades sociales fue el que evidenció requerir de mayor potenciación y trabajo, pero a través del trabajo en subgrupos, se tuvo la oportunidad de poner en práctica la capacidad de exponer ideas, negociar y tomar decisiones como grupo. Finalmente, el componente de procesamiento grupal se manejó con una retroalimentación externa del equipo facilitador y los subgrupos, sí se manifiestan orgullosos de su trabajo realizado. Es pertinente, después del análisis realizado, potenciar la autocrítica en los subgrupos, además de su sentimiento de logro por las tareas completadas.

A partir de estas conclusiones, se puede destacar que esta sistematización, es un aporte para las Ciencias de la Educación, en cuanto presenta una experiencia registrada de trabajo cooperativo, en un taller artístico, dirigido a personas en situación de discapacidad, donde se logran, en un espacio virtual, diferentes componentes de la cooperación. Encontrar prácticas desde lo virtual, que permitan el trabajo cooperativo y el desarrollo de habilidades sociales, podría implementar este tipo de prácticas en futuras intervenciones, lo que posibilitaría alcanzar una mayor cantidad de personas en situación de discapacidad, con un alcance territorial más amplio, de los proyectos dirigidos a esta población. 
Las conclusiones presentadas se basan en el análisis de la experiencia con un grupo de formación teatral que tenía ya varios años de estar funcionando de manera presencial, de manera que sería necesaria la réplica de este tipo de técnicas con grupos de menor experiencia para identificar si la adaptación a la virtualidad funciona de la misma manera.

Por esta razón, la primera línea de investigación, en las Ciencias de la Educación que se plantea a partir de esta experiencia, es la identificación y valoración de técnicas de trabajo virtual funcionales para el trabajo con personas en situación de discapacidad, con el objetivo de desarrollar habilidades sociales. La segunda línea de investigación que se esboza a partir de este trabajo, al tratarse de un grupo artístico, es la posibilidad de analizar el trabajo cooperativo en el proceso anual de montaje y ensayo de los productos artísticos del grupo, y no solo en actividades grupales por sesión, ya sea en el caso específico de este grupo en el ámbito teatral, como en otras disciplinas artísticas. Una tercera línea de investigación posible es explorar a mayor profundidad las percepciones de las personas en situación de discapacidad a trabajar de manera cooperativa, de modo que este trabajo pueda hacerse más atrayente y de interés para la población.

\section{REFERENCIAS}

ButerA, F. y BuChS, C. (2019). Social Interdependence and the Promotion of Cooperative Learning. En: K. Sassenberg, M. Vielk. Social Psychology in Action. Evidence-Based Intervertion from Theory to Practice. Gewerbestrasse: Spring.

Cedeño-Muñoz, J. C. y BARCiA-Briones, M. F. (2020). El aprendizaje cooperativo como estrategia educativa para estudiantes con discapacidades en bachillerato. Polo Del Conocimiento, 5(12), 616-636. https://doi.org/10.23857/pc.v5i12.2109

DeLIYORE VEGA, M. (2021). Networks as a social communication space for virtual education in students with disabilities in Costa Rica in pandemic times / Redes como espacio de comunicación para la educación virtual de estudiantes con discapacidad en Costa Rica en tiempos de pandemia. Historia y Comunicacion Social, 26, 75-85.

GuerRero, C. (2014). El ejercicio escénico como fenómeno propiciador del trabajo cooperativo y el aprendizaje significativo. Eutopía, 20, 72-77.

Herrada Valverde, R. I. \& Baños Navarro, R. (2018). Aprendizaje cooperativo a través de las nuevas tecnologías: Una revisión. @Tic. Revista D'Innovació Educativa, 20, 16. https://doi.org/10.7203/attic.20.11266

HuauYA, B. (2021). Las nuevas prácticas digitales de docentes de cursos artísticos en la educación superior en Latinoamérica, a raíz de la pandemia COVID-19: Aproximaciones y experiencias. Revista internacional de pedagogía e innovación educativa, 1(2), 29-40.

Huete, A. (2020). Pandemia y discapacidad . Lecciones a propósito del confinamiento. Revista Española de Discapacidad, 8(1), 203-207.

Hurtado Talavera, F. (2020). La Educación en Tiempos de Pandemia. CIEG Revista Arbitrada Del Centro de Investigación y Estudios Gerenciales, 44, 1-21. https://doi.org/10.52149/sp21/61.5

JoAN, R. (2018). Virtual Classroom: a Gift for Disabled Children. I-Manager's Journal on School Educational Technology, 14(2), 7-11. https://doi.org/10.26634/jsch.14.2.15100

Johnson, D. y JoHnson, F. (2014a). Joining together: Group theory and group skills (11th ed.). Pearson Education Limited.

JoHnson, D. W. y JoHnson, R. T. (2014b). Cooperative Learning in 21st Century. [Aprendizaje cooperativo en el siglo XXI]. Anales de Psicología, 30(3), 841-851. https://doi.org/10.6018/analesps.30.3.201241 
Jones, S. y DoolittLe, E. (2017). Social and Emotional Learning: Introducing the Issue. The Future of Children, 27(1), 3-11. Retrieved July 23, 2021, from http://www.jstor.org/stable/44219018

KORIS, R. y VUYLSTEKE, J. F. (2020). Mission (im)possible: developing students' international online business communication skills through virtual teamwork. In F. Helm \& A. Beaven (Eds.), Designing and implementing virtual exchange - a collection of case studies (Issue im, pp. 69-79). Research-publishing. net. https://doi.org/10.14705/rpnet.2020.45.1116

ORR, S. (2010). Collaborating or fighting for the marks? Students' experiences of group work assessment in the Creative Arts. Assessment and Evaluation in Higher Education, 35(3), 301-313. https://doi.org/10.1080/02602931003632357

Pellicano, E. y Stears, M. (2020). The hidden inequalities of COVID-19. Autism. https://doi.org/10.1177/1362361320927590

Pérez-Aldeguer, S. (2013). Effects of Collaborative Musical Theater on the Development of Social Competence. Pérez-Aldeguer, S.-118-Electronic Journal of Research in Educational Psychology, 11(1), $117-138$.

Peters, B.; Forlin, C.; Mclnerney, D. \& Maclean, R. (2013). Social Interaction and Cooperative Activities: Drawing Plans as a Means of Increasing Engagement for Children with ASD. International School of Whole Schooling, 9(2), 61-86.

Sandoval-Poveda, A. y Madriz-Bermúdez, L. (2020). Estrategias de acompañamiento virtual en un grupo de teatro inclusivo, durante tiempos de distanciamiento social. Virtual follow-up strategies in a Theatre Group in times of Social Distancing. Estratégias para o acompanhamento virtual em um grupo de teatro i. Innovaciones Educativas, 22 (N. ${ }^{\circ}$ Especial), 200-212.

https://doi.org/10.22458/ie.v22iespecial.3215

Schwarz Aguayo, L. (2011). Ventajas del aprendizaje cooperativo para el logro de conductas adaptativas de las personas con discapacidad intelectual. Educación, 17, 88-90. https://doi.org/10.33539/ educacion.2011.n17.1449

Segul, G. y Durán, D. (2011). Efectos del Aprendizaje Cooperativo en Niños Autistas. Revista Educación Inclusiva, 4(3), 12.

SILVA CRUZ, G. (2018). Estrategias de trabajo cooperativo y juegos grupales para favorecer las habilidades de interacción social en estudiantes con discapacidad intelectual y parálisis cerebral del 2do. grado de primaria, del Centro de Educación Básica Especial. N.o 005 Nuestro Señ [Instituto Pedagógico Nacional Monterrico]. In Estrategias Creativas Para Estudiantes Del IV Ciclo en el Nivel Primario.

SumArd, S. (2020). Adaptation To Emergency Remote Teaching: Pedagogical Strategy for Pre-Service Language Teachers Amid Covid-19 Pandemic. Turkish Online Journal of Distance Education, 22(2), 81-93.

Temprado Bernal, M. (2009). Ventajas del aprendizaje cooperativo para la socialización de los alumnos con necesidades educativas especiales. Revista Interuniversitaria de Formación del Profesorado, 65(23), 137-150. https://doi.org/10.2307/j.ctvn1tc6x.5 